Trauma Berufskrankh 2008 10 [Suppl 2]:203-207

DOI 10.1007/s10039-008-1367-z

Online publiziert: 11. April 2008

(c) Springer Medizin Verlag 2008

J. Schroeder-Printzen

Kanzlei Schroeder-Printzen Kaufmann \& Kollegen, Potsdam Hannover, Potsdam

\title{
Haftung, Regress und rechtliche Konsequenzen für den Arzt
}

Forschungseinrichtungen oder der medizinischen Wissenschaft, erforderlich ist vielmehr, dass sich diese Ergebnisse auch in der Praxis durchgesetzt haben [8]. Die Üblichkeit bzw. der Standard, der anzuwenden ist, gilt für den Zeitpunkt der Behandlung, sodass Veränderungen in der medizinischen Wirklichkeit nach diesem Zeitpunkt unberücksichtigt bleiben [16].

\section{Abgrenzungsfragen innerhalb der Unfallversicherung}

Eine objektiv unrichtige Diagnose stellt nicht unmittelbar einen Behandlungsfehler dar, vielmehr muss die Erkennbarkeit der Unrichtigkeit der objektiven Diagnose hinzutreten [17], daher ist die Rechtsprechung bei der Annahme eines Diagnosefehlers eher zurückhaltend [9]. Ein Diagnoseirrtum ist dann lediglich vorwerfbar,

- wenn medizinische Symptome vorliegen, die für eine bestimmte Krankheit kennzeichnend sind, und vom Arzt nicht ausreichend berücksichtigt werden, oder

- wenn der Arzt ohne vorwerfbare Fehlinterpretation von Befunden eine objektive unrichtige Diagnose stellt, weil er eine notwendige Befunderhebung unterlassen hat $[8,9,17]$.

\section{Behandlungsfehler im engeren Sinne}

Er ist immer eine unzulässige Abweichung nach unten vom allgemein anerkannten Standard der Behandlung des Patienten [9, 16]. Dies bedeutet gleichzeitig, nicht entscheidend sind die Ergebnisse von
Beim D-Arzt ist in der Rechtsprechung [13] anerkannt, dass für den Diagnosefehler und, wenn hierauf aufbauend, eine fehlerhafte Entscheidung über die weitere Behandlung - besondere Heilbehandlung nach $\$ 34$ SGB VII - nicht der D-Arzt unmittelbar in die Haftung zu nehmen ist. Vielmehr übt er zu diesem Zeitpunkt eine hoheitliche Tätigkeit aus, die dazu führt, dass die jeweilige Berufsgenossenschaft nach den Grundsätzen der Amtshaftung nach $\$ 839$ BGB i.V.m. Artikel 34 GG in Anspruch genommen werden muss. Diese kann dann prüfen, ob sie gegenüber dem jeweiligen Arzt Regressansprüche geltend macht.

Beim H-Arzt ist die Rechtsprechung noch nicht so eindeutig. Während das LG Karlsruhe die oben ausgeführten Grundsätze auch für den D-Arzt anwenden wollte, gelangte das OLG Karlsruhe [10] als Berufungsinstanz zu dem Ergebnis, die Anwendung der Haftung der Berufsgenossenschaft sei zu verneinen. Es bleibt nunmehr abzuwarten, wie der BGH diese Frage beurteilen wird, wobei grundsätzlich vieles dafür sprechen sollte, dass auch hier die Abgrenzung wie beim DArzt übernommen wird.

Ist dem Arzt bei der weitergehenden Behandlung des in der BG versicherten Patienten ein Behandlungsfehler anzulasten, also eine fehlerhafte Behandlung, haftet er dem Patienten sowohl aus dem unmittelbar abgeschlossenen Behandlungsvertrag als auch aus unerlaubter Handlung $[13,16]$. Letzteres ergibt sich aus der Tatsache, dass der Patient nur wirksam in eine Behandlung lege artis einwilligt, eine nicht lege artis durchgeführte Behandlung ist in aller Regel nicht einwilligungsfähig.

\section{Haftungsumfang gegenüber dem Patienten}

Er ist relativ weit, der Arzt hat den Patienten nach $\$ \$ 249 \mathrm{ff}$. BGB so zu stellen, wie er stehen würde, wenn das schädigende Ereignis nicht eingetreten wäre; ferner besteht ein Schmerzensgeldanspruch nach \$253 Abs. 2 BGB. Hierbei erhält der Patient im Wesentlichen zunächst ein einmaliges Schmerzensgeld, im Ausnahmefall in besonders schwerwiegenden Fällen auch eine Schmerzensgeldrente [5].

Des Weiteren hat er Anspruch auf Verdienstausfall, wenn er aufgrund des ärztlichen Behandlungsfehlers weniger Geld verdient als er vorher verdient hat. Sollte ferner eine weitere Tätigkeit im Haushalt aufgrund des Behandlungsfehlers nicht oder nur eingeschränkt möglich sein, können auch Schadensersatzpflichten im Zusammenhang mit der Haushaltsführung auftreten.

Voraussetzung für alle Fälle ist jedoch, dass die eingetretene Beeinträchtigung 
beim Patienten kausal durch den Behandlungsfehler des Arztes verursacht wurde $[8,16]$. Bei den Kausalfragen tauchen in der Praxis häufig viele medizinische Fragen auf, die durch den Sachverständigen darzustellen sind. Liegen Beeinträchtigungen vor, die nicht ursächlich zum Behandlungsfehler sind, ist eine Haftung in diesem Zusammenhang zu verneinen [16].

\section{Haftung gegenüber den Sozialversicherungsträgern}

Sozialversicherungsträger wenden üblicherweise eine Vielzahl von Kosten auf, seien es beispielsweise im Bereich der gesetzlichen Krankenversicherung die Gewährung von Krankengeld ( $\$$ \$4ff. SGB $\mathrm{V}$ ) und Behandlungskosten ( $\$ 27 \mathrm{ff}$ SGB V); im Bereich der Unfallversicherung die Gewährung von Heilbehandlung (\$\$27ff. SGB VII), Verletztengeld ( $\$ \$ 45$ ff. SGB VII) u.v.m.

Auf Grundlage von \$116 SGB X werden kraft gesetzlicher Anordnung - cessio legis - die Ansprüche auf Ersatz der Kosten für die entsprechenden kausal sich aus dem Behandlungsfehler ergebenden Behandlungskosten auf den entsprechenden Sozialversicherungsträger übergeleitet, hier gelten die gleichen Kausalitätsgrundsätze wie beim Patienten. Es bedarf keiner Abtretung von Schadensersatzansprüchen, vielmehr geht der Anspruch automatisch über, und der Sozialversicherungsträger macht dann auch in aller Regel entsprechende Schadensersatzansprüche gegenüber dem schädigenden Arzt bzw. dessen Haftpflichtversicherer geltend.

\section{Haftung gegenüber der privaten Versicherung}

Sofern nicht die gesetzlichen Sozialversicherungsträger leistungspflichtig sind, sondern ein privater Versicherer beim $\mathrm{Pa}$ tienten die entsprechenden Kosten übernimmt, ist für den gesetzlichen Forderungsübergang nicht der \$116 SG X, sondern der $\$ 86$ VVG in der ab 01.01.2008 geltenden neuen Fassung bzw. der $\$ 67$ VVG in der bis dahin geltenden alter Fassung maßgebend. Diese Vorschriften leiten aber auch in diesem Bereich die Forderungen entsprechend auf den priva- ten Krankenversicherer/Pflegeversicherer über. Die Kausalitätsanforderungen sind in diesem Zusammenhang die gleichen wie bei \$116 SGB X und bei der Haftung dem Patienten gegenüber.

\section{Verhältnis zum Haftpflichtversicherer}

In diesem Zusammenhang sind 2 Probleme zu trennen; einmal präventive Maßnahmen und zum anderen die richtigen Verhaltensweisen im Schadensfall.

Innerhalb des präventiven Bereichs ist regelmäßig zu kontrollieren, ob die Haftpflichtversicherung den allgemeinen Bedürfnissen der Praxis noch entspricht [11]. Es kann durchaus sein, dass sich das Spektrum der ersten Tätigkeit ändert, ohne dass die Versicherungsverträge angepasst werden. Das hat jedoch zur Konsequenz, dass bei einem nicht mehr mitversicherten Risiko im Schadensfall keinerlei Deckungsschutz besteht ( $\$ \$ 23,26 \mathrm{VVG})$.

Im Schadensfall selbst ist der Versicherer berechtigt, den Schadensfall zu regulieren. Hierfür ist es erforderlich, dass der Arzt diesen von einem möglichen Schadensfall unverzüglich in Kenntnis setzt, was sich aus \$30 VVG ergibt. Des Weiteren ist es dem Arzt nicht gestattet, bereits eine Entscheidung über das Anerkennen einer Haftung dem Grunde nach auszusprechen, da ansonsten der Versicherungsschutz gefährdet ist.

\section{Strafrechtliche Gesichtspunkte}

Der Arzt bewegt sich bei seiner ärztlichen Tätigkeit immer in einem strafrechtlich relevanten Bereich. Hinsichtlich der Behandlung von Patienten ist regelmäßig an Körperverletzungsdelikte ( $\$ \$ 223 \mathrm{ff}$. StGB) oder an die fahrlässige Tötung ( $\$ 222$ $\mathrm{StGB})$ sowie an die unterlassene Hilfeleistung ( \$323c StGB) zu denken. Speziell im Verhältnis zum Patienten ist noch zusätzlich die strafrechtlich bewährte Verletzung der Schweigepflicht ( $\$ 201$ StGB) zu beachten $[14,18]$.

Hinsichtlich der Abrechnung und der dort vorsätzlich vorgenommenen unrichtigen Abrechnungen sind als Straftatbestände an den Betrug ( $\$ 263$ StGB), die Urkundsdelikte ( $\$ \$ 267 f f$. StGB) sowie an die Untreue (\$266 StGB) zu denken. Soll- ten im Einzelfall noch Amtsträger beteiligt sein, sind zusätzlich die $\$ \$ 331 \mathrm{ff}$ StGB zu beachten [14, 18].

Innerhalb des Arztstrafrechts sollten im Regelfall nur mit Hilfe eines Rechtsanwalts etwaige Aussagen als Beschuldigter gegenüber der Polizei oder gegenüber der Staatsanwaltschaft gemacht werden. Es bietet sich auch an, generell schon sehr frühzeitig einen Anwalt in das Verfahren einzubinden, da dieser über die notwendige Fachkunde im Umgang mit der Staatsanwaltschaft verfügt.

\section{Verhalten gegenüber der Polizei und der Staatsanwaltschaft}

Sollen bei einer staatsanwaltschaftlichen Durchsuchung Unterlagen beschlagnahmt werden, ist der Beschlagnahme nicht zuzustimmen. Ansonsten sollte man sich grundsätzlich kooperativ zeigen, da sonst auch die Kooperationswilligkeit der Staatsanwaltschaft eingeschränkt ist. Dies kann zur Konsequenz haben, dass keinerlei Unterlagen mehr in der Praxis sind, um Behandlungen ordnungsgemäß durchführen zu können [18].

Bei einer Durchsuchsuchung der Praxis- und/oder Privaträume sollte darauf geachtet werden, dass inhaltliche Gespräche mit der Polizei oder den Beamten der Staatsanwaltschaft nicht geführt werden, da deren Inhalt durch einen Aktenvermerk Bestandteil der Ermittlungsakte wird [18].

Sollten insbesondere Krankenunterlagen beschlagnahmt werden, ist $\mathrm{zu}$ empfehlen, diese $\mathrm{zu}$ fotokopieren, damit man auch selbst über eine ausreichende Informationsgrundlage verfügt, um ggf. inhaltlich argumentieren zu können. Gleiches gilt bei der Beschlagnahme von Abrechnungsunterlagen wegen Abrechnungsbetrugs. Diese Unterlagen sind für eine weitere Verteidigung zwingend erforderliche Grundlagen.

Speziell bei einer Beschlagnahme im Ermittlungsverfahren wegen eines Behandlungsfehlers ist gleichfalls der Haftpflichtversicherer unverzüglich hiervon in Kenntnis zu setzen [11] und mit diesem abzustimmen, ob evtl. schon jetzt frühzeitig ein Privatgutachten in Auftrag gegeben werden soll, in welchem geprüft 
wird, ob tatsächlich ein Behandlungsfehler vorliegt.

\section{Einstellung des Strafverfahrens gegen Zahlung einer Geldbuße}

Da insbesondere im Arztstrafrecht ein erheblicher Ermittlungsaufwand auch für die Staatsanwaltschaft besteht, stellt sich relativ schnell die Frage, ob nicht ein Einverständnis zur Einstellung des Ermittlungsverfahrens ohne Zahlung einer Geldbuße nach $\$ 153$ StPO oder eine Einstellung gegen Zahlung einer Geldbuße nach \$153a StPO ausgesprochen werden sollte, sofern nicht eine Einstellung mangels Tatverdachts nach $\$ 170$ Abs. 2 StPO in Betracht kommt.

Die Einstellung nach \$153a StPO bedarf der Zustimmung des Beschuldigten. Sie ist eine für die Praxis nicht zu unterschätzende Vorschrift, mit der die Möglichkeit besteht, ohne vollständige Ermittlung des Sachverhaltes das Ermittlungsverfahren einzustellen. Wird eine entsprechende Zustimmungserklärung abgegeben, gesteht der Arzt kein Eingeständnis ein, tatsächlich falsch abgerechnet zu haben, es gilt für ihn vielmehr dann nach wie vor die volle strafrechtliche Unschuldsvermutung [18]. Dies kann insbesondere innerhalb der vertragsarztrechtlichen Betrachtungsweise im Zusammenhang mit dem Abrechnungsbetrug oder auch für berufsgerichtliche Maßnahmen von unschätzbarer Bedeutung sein.

Des Weiteren ist abzuwägen, ob man tatsächlich die Durchführung einer Hauptverhandlung wünscht. Hier spielen weniger juristische als vielmehr rein praktische Überlegungen eine Rolle. Dabei ist zunächst zu berücksichtigen, dass die Durchführung einer Hauptverhandlung den Arzt nicht nur Zeit, sondern auch erhebliche Geldbeträge kostet, denn üblicherweise werden solche Hauptverhandlungsverfahren aus anwaltlicher Sicht nur unter Zugrundelegung einer Honorarvereinbarung durchgeführt, mit der Konsequenz, dass auch im Fall eines Freispruchs nicht sämtliche Kosten für die Vertretung durch einen Anwalt erstattet werden [18].

Auch ist zu berücksichtigen, dass die Durchführung der Hauptverhandlung zwingend die Anwesenheit des Arztes voraussetzt, er kann für diese Zeit keiner- lei weitere ärztliche Tätigkeit ausüben, was gleichfalls mit entsprechenden wirtschaftlichen Verlusten einhergeht [18].

Des Weiteren ist zu berücksichtigen, dass nach Nr. 26 Anordnung über Mitteilungen in Strafsachen Abschriften der Anklageschrift sowohl der Approbationsbehörde als auch der Ärztekammer zur Verfügung gestellt werden. Dies kann dann noch weitere - für den Arzt zeitlich aufwändige - Verwaltungsverfahren nach sich ziehen, die mit einer Einstellung nach \$153a StPO vermieden werden würden. Die Ärztekammern erfahren in einem solchen Fall von der Staatsanwaltschaft nichts hinsichtlich des Ermittlungsverfahrens [18].

\section{Berufsrecht}

Es ist reines Landesrecht, es orientiert sich jedoch an der Musterberufsordnung, auf die vorliegend Bezug genommen wird. Des Weiteren ist berufsrechtlich zu berücksichtigen, dass die Heilberufsgesetze der Länder die Möglichkeit der Ahndung berufsrechtlicher Pflichtverstößen vorsehen. Dieses Landesrecht ist in allen Bundesländern ziemlich ähnlich, sodass hierzu auch allgemeine Ausführungen möglich sind.

Sowohl der Behandlungsfehler als auch der Abrechnungsbetrug stellen als solches Verletzungen der Berufsordnung dar, was sich hinsichtlich des Behandlungsfehlers aus $\mathrm{C} \mathrm{Nr} .2 \mathrm{MBO}$ und hinsichtlich des Abrechnungsbetruges aus $\$_{2} \mathrm{Abs}$. $2 \mathrm{MBO}$ ergibt [1].

Die Heilberufsgesetze der jeweiligen Bundesländer sehen bei Berufspflichtverletzungen grundsätzlich folgende Maßnahmen vor [12]:

$$
\begin{aligned}
& \text { - Verwarnung } \\
& \text { - Verweis } \\
& \text { - Geldbuße bis 50.000,00 EUR } \\
& \text { - Entziehung des aktiven und passiven } \\
& \text { Kammerwahlrechts } \\
& \text { - Feststellung der Unwürdigkeit der Be- } \\
& \text { rufsausübung }
\end{aligned}
$$

Auch dieses berufsgerichtliche Verfahren ist langwierig und kann den Arzt viel Zeit kosten. Es wird von den Verwaltungsgerichten geführt.
Trauma Berufskrankh 2008 · 10

[Suppl 2]:203-207

DOI 10.1007/s10039-008-1367-z

(C) Springer Medizin Verlag 2008

\section{J. Schroeder-Printzen}

\section{Haftung, Regress und rechtliche Konsequenzen für den Arzt}

\section{Zusammenfassung}

Die Konsequenzen eines fehlerhaften Verhaltens eines Arztes bei der Ausübung seiner ärztlichen Tätigkeit sind vielfältig. Beim Behandlungsfehler ist die zivil- und strafrechtliche Haftung von besonderer Bedeutung. Hier ist eine enge Zusammenarbeit mit dem Haftpflichtversicherer erforderlich. Bei strafrechtlich relevanten Verfahren, sei es wegen eines Behandlungsfehlers oder wegen Abrechnungsbetrugs, ist frühzeitig die Einschaltung eines Anwalts erforderlich. Bei einem Abrechnungsbetrug ist immer die vertragsärztliche Zulassung oder die Zulassung als D- oder H-Arzt in Gefahr. Ferner ist mit nicht unerheblichen Honorarrückforderungen zu rechnen. Sowohl wegen des Behandlungsfehlers als auch bei Abrechnungsbetrug ist mit einem berufsgerichtlichen Verfahren zu rechnen oder auch an das Ruhen bzw. die Entziehung der Approbation zu denken.

\section{Schlüsselwörter}

Behandlungsfehler · Abrechnungsbetrug . Approbation - Berufsgerichtliches Verfahren . Vertragsarztrecht

\section{Liability, recourse and legal consequences for the doctor}

\section{Abstract}

The consequences of an incorrect action by a doctor while exercising his or her medical practice are manifold. For errors in treatment, civil and criminal liability is especially important. Here a close cooperation with the liability insurer is essential. For criminal proceedings, whether for errors in treatment or for fraudulent invoicing, retaining a lawyer early on is necessary. In case of fraudulent invoicing, there is always the danger of losing the contract status with the insurance company. Furthermore, the return of not insubstantial medical fees is to be anticipated. In the case of errors treatment as well as fraudulent invoicing, professional conduct proceedings are to be expected, and the suspension or even loss of accreditation is possible.

\section{Keywords}

Treatment error · Fraudulent invoicing · Licensure - Professional conduct investigation . Doctor-insurance contract law 


\section{Vertragsarztrecht}

Diesbezüglich kommt es beim Abrechnungsbetrug gleichfalls zu einer Vielzahl von Problemen, die der Arzt zu bewältigen hat. In der Praxis spielen hier Behandlungsfehler keine Rolle, obwohl man auch in diesem Zusammenhang davon ausgehen kann, dass eine Pflichtverletzung des Arztes vorlag, da er auch vertragsarztrechtlich verpflichtet ist, den allgemeinen ärztlichen Standard einzuhalten.

\section{Honoraranspruch}

Speziell bei unrichtiger Abrechnung innerhalb der vertragsärztlichen Versorgung ist hinsichtlich des Abrechnungsbetruges zu berücksichtigen, dass aufgrund einer vorsätzlich falsch abgegebenen Abrechnungssammelerklärung die Kassenärztliche Vereinigung berechtigt ist, den Honoraranspruch des Arztes neu zu schätzen [15]. Dabei räumt das Bundessozialgericht [2] der Kassenärztlichen Vereinigung ein breites Schätzungsermessen ein, das im Regelfall nach wie vor noch als richtig ausgeübt gilt, wenn der Arzt auf den Fachgruppendurchschnitt gekürzt wird. Diese Entscheidung betraf einen Zeitraum, in dem Honorarbudgetierungen auf Grundlage der Honorarverteilungsmaßstäbe bzw. des EBM keinerlei Rolle spielten. Ob dies unter Beachtung der nunmehr geltenden Budgetierungen auch innerhalb des EBM bzw. des Honorarverteilungsvertrages noch so uneingeschränkt zur Anwendung gelangen kann, erscheint fraglich. Argumentativ wird man sich mit der Frage auseinandersetzen zu haben, ob unter Berücksichtigung der unrichtig abgerechneten Gebührenziffern tatsächlich ein geringerer Honoraranspruch existiert oder ob sich die Abrechnungsfehler auf den gesamten Honoraranspruch des Arztes nicht auswirken, da nach Bereinigung der Fehlerquote nach wie vor derselbe Honoraranspruch besteht.

Sollte jedoch tatsächlich Honorar zurückgefordert werden, haben weder Widerspruch noch die Klage aufschiebende Wirkung, was sich aus $\$ 85$ Abs. 4 Satz 9 SGB V ergibt. Das hat zur Folge, dass das zurückgeforderte Honorar sofort zurückgezahlt werden muss. Um dies zu verhin- dern, besteht die Möglichkeit, nach $\$ 86 \mathrm{~b}$ SGG einen Antrag zu stellen, dass die aufschiebende Wirkung des Widerspruchs bzw. der Klage angeordnet wird. Hierbei ist dann zu erläutern, warum der Honoraraufhebungs- und -rückforderungsbescheid rechtswidrig ist. Des Weiteren sind die wirtschaftlichen Konsequenzen auf die Praxis darzustellen. Wenn die Liquidität der Praxis trotz der Rückforderung noch ausreichend ist, wird häufig bereits aus diesem Gesichtspunkt heraus - ohne Prüfung der materiellen Rechtmäßigkeit des Bescheides - die Anordnung der aufschiebenden Wirkung des Widerspruchs/ der Klage abgelehnt.

Sollte der Arzt eine Untreue begangen haben, wird er Schadensersatzansprüchen der geschädigten Krankenkassen entgegensehen müssen.

\section{Auswirkungen auf die vertragsärztliche Tätigkeit}

Der Abrechnungsbetrug stellt nach gefestigter Rechtsprechung des BSG eine gröbliche Pflichtverletzung dar, sodass unter Berufung auf $\$ 95$ Abs. 6 Satz 1 SGB $\mathrm{V}$ die Zulassung entzogen werden kann, wobei seit 01.01.2007 auch die Möglichkeit existiert, diese nur hälftig zu entziehen ( $\$ 95$ Abs. 6 Satz 2 SGB V) [6]. Der Berufungsausschuss hat zusätzlich unter Berufung auf $\$ 97$ Abs. 4 SGB V die Möglichkeit, die sofortige Vollziehung der $\mathrm{Zu}$ lassungsentziehung anzuordnen. Dies ist nur dann zulässig, wenn ein besonderes öffentliches Interesse hierfür besteht. Dies aber ist in der Regel bereits zu bejahen, wenn der Arzt in unmittelbarer Vergangenheit vor der Entscheidung der Zulassungsgremien falsch abgerechnet hat.

\section{Berufsgenossenschaft}

Sollte speziell bei der berufsgenossenschaftlichen Tätigkeit ein Abrechnungsbetrug vorgekommen sein, besteht die Gefahr des Verlustes der Beteiligung als D- oder als H-Arzt im Sinne von $\$_{34}$ SGB VII. Dies geschieht im Wege des Verwaltungsaktes durch den zuständigen Landesverband der gewerblichen Berufsgenossenschaften [3]. Die Berufsgenossenschaft kann hinsichtlich der Entziehung dieser Stellung die sofortige Vollziehung nach $\$ 86 \mathrm{a}$ Abs. 2 Nr. 5 SGG anordnen, sofern ein besonderes öffentliches Interesse besteht.

\section{Approbationsrecht}

Auch approbationsrechtlich kann ein strafrechtlich relevantes Verhalten des Arztes erhebliche Konsequenzen haben. Dies gilt nicht nur im Falle des Abrechnungsbetruges, sondern auch wenn Behandlungsfehler vorgekommen sind [7].

Das Ruhen der Approbation nach $\$ 6$ Abs. 1 Nr. 1 BÄO kann dann angeordnet werden, wenn gegen den Arzt wegen des Verdachts einer Straftat, aus der sich seine Unwürdigkeit oder Unzuverlässigkeit zur Ausübung des ärztlichen Berufs ergeben kann, ein Strafverfahren eingeleitet wird.

Das Ruhen hat zum Inhalt, dass die Stellung als Arztes vorläufig verlustig wird und keine Berechtigung mehr besteht, überhaupt ärztlich tätig zu sein [7]. Beim Ruhen der Approbation kann die sofortige Vollziehung nach $\$ 80$ Abs. 2 Satz $1 \mathrm{Nr} .4 \mathrm{VwGO}$ angeordnet werden. Hierbei ist aber zu beachten, dass dies einen erheblicher Eingriff in die Berufsfreiheit des Arztes aus Art. 12 GG darstellt, der einer ausführlichen Begründung bedarf. Es muss dargelegt werden, warum zum Schutz wichtiger Gemeinschaftsgüter eine sofortige Vollziehung erforderlich ist. Hierfür ist es notwendig, dass die Gründe weiter reichen als die das Ruhen der Approbation rechtfertigenden [4].

Ferner besteht die Möglichkeit, insbesondere bei einer rechtskräftigen Verurteilung innerhalb eines Strafverfahrens, den Widerruf der Approbation auszusprechen, was sich aus $\$_{5}$ Abs. 2 BÄO ergibt. Konsequenz hieraus sind der endgültige Verlust der Stellung als Arzt und auch der endgültige Verlust, ärztlich tätig zu sein. Auch hinsichtlich des Widerrufs der Approbation besteht die Möglichkeit der Anordnung der sofortigen Vollziehung.

\section{Korrespondenzadresse}

\section{J. Schroeder-Printzen}

Kanzlei Schroeder-Printzen Kaufmann \&

Kollegen, Potsdam Hannover

Kurfürstenstraße 31, 14467 Potsdam

sekretariat.sp@spkt.de

Interessenkonflikt. Der korrespondierende Autor gibt an, dass kein Interessenkonflikt besteht. 


\section{Literatur}

1. Buchner R, Jäkel C (2005) Berufsrecht der Heilberufe. In: Stellpflug M, Meier S, Tadayon A (Hrsg) Handbuch Medizinrecht. Müller/MedizinRecht.de, Heidelberg, B1000

2. Bundessozialgericht (1997) Urteil vom 17.09.1997 - 6 RKa 86/95 - Sozialrecht 3-5550 §35 EKV-Ärzte Nr. 1. Bundessozialgericht, Kassel

3. Bundessozialgericht (2006) Urteil vom 05.09 .2006 - B 2 U 8/05 R - Sozialrecht 4-2700 \$34 Nr. 1. Bundessozialgericht, Kassel

4. Bundesverfassungsgericht (2003) Beschluss vom 24.10.2003 - 1 BvR 1594/03. Neue Jurist Wochenschr 56: 3618-3619

5. Hacks S, Ring A, Böhm P (2008) Schmerzensgeldbeträge. Deutscher Anwaltverlag, Bonn

6. Hartmannsgruber K, Schroeder-Printzen J (2008) Vertragsarztrecht. In: Ratzel R, Luxenburger $B$ (Hrsg) Handbuch Medizinrecht. Deutscher Anwaltverlag, Bonn, §7

7. Jäkel C (2007) Berufszugangsregelungen für Ärzte: Bundesärzteordnung und Approbationsordnung. In: Stellpflug M, Meier S, Tadayon A (Hrsg) Handbuch Medizinrecht. Müller/MedizinRecht.de, Heidelberg, B2000

8. Kaiser S (2008) Arzthaftungsrecht. In: Ratzel R, Luxenburger B (Hrsg) Handbuch Medizinrecht. Deutscher Anwaltverlag, Bonn, §12

9. Müller G (2004) Macht und Grenzen ärztlichen Handelns. Gesundheitsrecht 3: 257-266

10. Oberlandesgericht Karlsruhe (2007) Urteil vom 14.11.2007 - 7 U 101/06. OLG Karlsruhe

11. Ratzel $R$ (2008) Haftpflichtversicherungsrecht. In: Ratzel R, Luxemburger B (Hrsg) Handbuch Medizinrecht. Deutscher Anwaltverlag, Bonn, §13

12. Rehborn M (2004) Berufsgerichtliche Verfahren gegen Ärzte - grundlegende Rechtsfragen. Gesundheitsrecht 3: 170-176

13. Schleswig-Holsteinisches Oberlandesgericht (2007) Urteil vom 02.03.2007 - 4 U 22/06. Gesundheitsrecht 6: 469-471

14. Schmidt J, Giring J (2008) Arztstrafrecht. In: Ratzel R, Luxemburger B (Hrsg) Handbuch Medizinrecht. Deutscher Anwaltverlag, Bonn, §14

15. Schroeder-Printzen J (2005) Einheitlicher Bewertungsmaßstab und Abrechnung. In: Stellpflug M, Meier S, Tadayon A (Hrsg) Handbuch Medizinrecht. Müller/MedizinRecht.de, Heidelberg, D2000

16. Steffen E, Pauge B (2006) Arzthaftungsrecht. RWS, Köln

17. Strücker-Pitz H (2007) Zivilrechtliche Haftung: Behandlungsverschulden. In: Stellpflug M, Meier S, Tadayon A (Hrsg) Handbuch Medizinrecht. Müller/ MedizinRecht.de, Heidelberg, F2000

18. Weimann A, Funcke-Auffermann N (2007) Strafrechtliche Haftung. In: Stellpflug M, Meier S, Tadayon A (Hrsg) Handbuch Medizinrecht. Müller/MedizinRecht.de, Heidelberg, F5000 